\title{
Performance Analysis of Multicast MAC Protocol for Multichannel Dual Bus Networks
}

\author{
Shiann-Tsong Sheu ${ }^{1}$ and Jenhui Chen ${ }^{2}$ \\ 1 Department of Electrical Engineering, \\ Tamkang University, Tamsui, Taipei, Taiwan 25137, R.O.C. \\ stsheu@ee.tku.edu.tw \\ 2 Department of Computer Science and Information Engineering, \\ Tamkang University, Tamsui, Taipei, Taiwan 25137, R.O.C. \\ jenhui@cs.tku.edu.tw
}

\begin{abstract}
The WDM-based multichannel dual bus networks (MCDBN) for high-speed LAN/MAN was proposed in the few years. When a station requests to connect to another, the transmitter of source station and the receiver of destination station must listen to a same channel (wavelength). The problem of using minimal wavelengths for a set of requests on MCDBN had been proved as NP-hard. Intuitively, the problem of providing the multicast services over MCDBN is more complicated. In this paper, we will analyze the random approach (RAND) and the Best Effort Multicasting Approach (BEMA) which was proposed for multicast packet transmissions on MCDBN. The derived analysis results are very close to the simulation results.
\end{abstract}

\section{Introduction}

The dual bus topology for high-speed LAN/MAN was proposed in the few years 1112. One of the major characteristics of such multi-access networks is that it provides a low access delay even when the number of connecting nodes is large. Several distributed protocols on optical bus networks, such as distributed queue dual bus (DQDB) [11, had been proposed. However, the maximum network capacity is still bounded due to only one channel is available on the optical fiber [3]. For solving this problem, the Wavelength Division Multiplexing (WDM) [2, 14[15] is used to support a large number of transmission channels in parallel. Several multi-channel photonic star/bus/ring networks with unicasting protocols had been reported in which either node has tunable-transmitter(s) (TT) and fixed-receiver(s) (FR) [13/5]6] or node has tunable-transmitter(s) (TT) and tunable-receiver(s) (TR) [8913]. In paper [10], the wavelength/receiver assignment problem (WRAP) of unicast services is defined on a multi-channel dual bus network (MCDBN) to assign a transmission wavelength and a receiver for each of the request such that the network throughput is maximized and the number of assigned wavelengths is also minimized. The paper also proves the WRAP is NP-hard. Obviously, the problem of providing multicast services over MCDBN is more complicated than that of supporting unicast services. For example, when 
a node requests to transmit a packet to several multicast members, if it can not find a channel which is listen by all these members, it must transmit the packets over several different channels to complete this transmission. Therefore, how to minimize the number of transmission times of a same packet is also a complicated problem. So far, only few protocols have been proposed for multicast services on MCDBN. In paper [16], we had proposed a simple and efficient protocol, named as the Best Effort Multicasting Approach (BEMA), for multicast services. The BEMA tries to minimize the transmission times and maximize the channel utilization. In this paper, we will precisely analyze the average transmission times of the proposed BEMA.

The rest of this paper is organized as follows. The architecture of WDMbased multi-channel dual bus network (MCDBN) is introduced in Section 2, In Section 3, we present the BEMA multicasting protocol. The detailed analyses of the BEMA and random approach are addressed in Section 4 . The simulation models and simulation results are reported in section 5. Finally, some conclusion remarks are given in Section [6.

\section{The Architecture of MCDBN}

The architecture of a MCDBN consists of two contra-directional optical buses, which allow full duplex communications between any two nodes as shown in Fig. 11 The network contains $\mathrm{N}$ stations. Each station has $m$ TRs and $n$ TRs. The two buses are called Bus A and Bus B, respectively. For each bus, the entire bandwidth is divided into $C+1$ channels $\left\{\lambda_{0}, \lambda_{1}, \lambda_{2}, \ldots, \lambda_{C}\right\}$, where channel $\lambda_{0}$ is the dedicated control channel and others are data channels. For each channel, fixed length slots are generated by the headend station (also called slot generator) periodically, passed to the downstream stations and is terminated by the terminator.

\section{The BEMA Multicasting Protocol}

In this section, we will briefly introduce the proposed BEMA multicasting protocol for the MCDBN.

The data slot format of the MCDBN is the same as that of the DQDB. The control signals are arranged in the payload field. Fig. 2 shows the format of a control slot. A control slot includes three major fields: the multicast request field REQ_MULTICAST, the BROADCAST field and the RESERVATION field. The REQ_MULTICAST field contains four subfields: SRC, MDST, LEN and SWAVE. The BROADCAST field contains SRC and UWAVE subfields. It is used for each station to inform all stations the wavelength usage information. The $C$-bit RESERVATION field, one bit for each wavelength, carries the requests of slots issued from the downstream stations. Based on this protocol, within a cycle time, each station can maintain the newly and correctly traffic information of all stations in a traffic status matrix (TSM). The TSM is defined as follows: 
Headend

Terminator

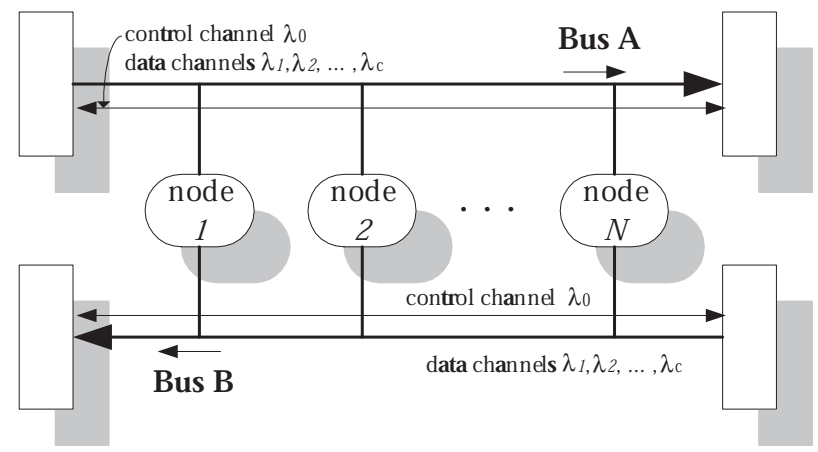

Terminator

Headend

Fig. 1. The construction of a WDM-based dual bus networks.

- Traffic Status Matrix: $M=\{t(i, j)\}_{N \times N}, i, j=1,2, \ldots, N$, where $t(i, j)=$ $k, k \in\{1, \ldots, C\}$. Each element $t(i, j)=k(k>0)$ indicates that wavelength $\lambda_{k}$ is used for station $i$ to transmit packet to station $j$, and $t(i, j)=0$ otherwise.

For illustration, consider the example shown in Fig. 3. The Traffic Status Matrix of stations 8 is :

$$
M_{8}=\left(\begin{array}{cccccccc}
0 & 2 & 0 & 0 & 0 & 0 & 0 & 0 \\
0 & 0 & 1 & 2 & 0 & 0 & 0 & 0 \\
0 & 0 & 0 & 1 & 0 & 3 & 0 & 0 \\
0 & 0 & 0 & 0 & 2 & 0 & 0 & 1 \\
0 & 0 & 0 & 0 & 0 & 2 & 0 & 0 \\
0 & 0 & 0 & 0 & 0 & 0 & 2 & 3 \\
0 & 0 & 0 & 0 & 0 & 0 & 0 & 0 \\
0 & 0 & 0 & 0 & 0 & 0 & 0 & 0
\end{array}\right)
$$

In [10], an efficient load balancing wavelength assignment algorithm (LBWAA) had been proposed for the unicast traffic on multi-channel dual bus network. However, for a multicast packet, the number of transmission times for it may be larger than one because that the number of tunable receivers equipped in each station is small. In detail, it is possible that all TRs of multicast members are assigned to listen on different channels. As a result, a multicast packet may be required to transmit to all multicast members by different wavelengths. For example, if there are any two multicast members do not tune their receivers into a same channel at that time, the multicast packet must be transmitted twice (one for each station). This causes the bandwidth wasted seriously. In the next paragraphs, we will briefly describe the BEMA protocol. 

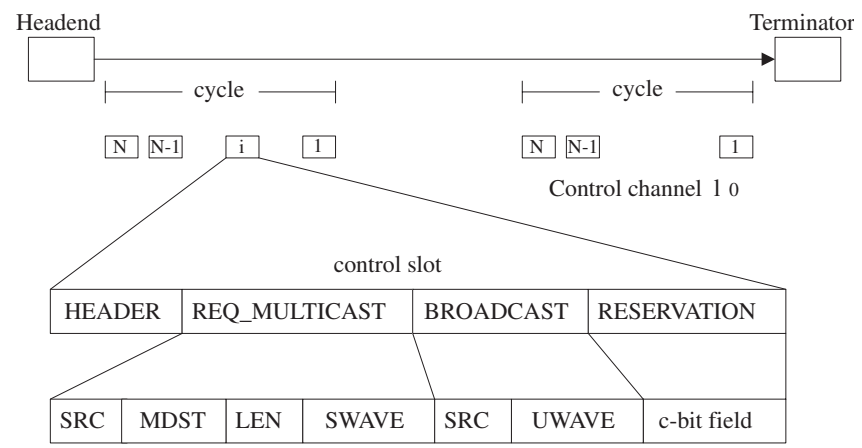

SRC : source station.

MDST : multicast destination stations.

LEN : message length.

SWAVE : selected wavelength

UWAVE : using wavelength.

Fig. 2. The slot format on control channel.

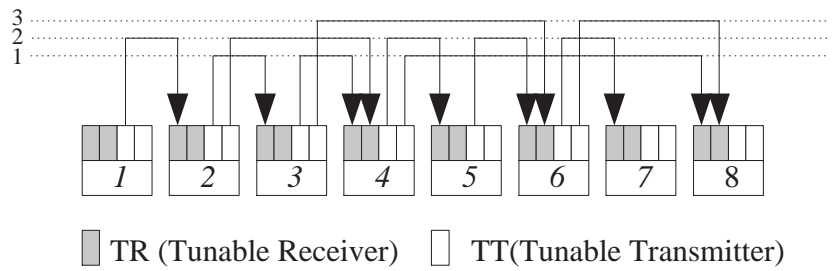

Fig. 3. An example of $\mathrm{MCDBN}$ for $\mathrm{C}=3, \mathrm{~T}=2, \mathrm{R}=2$ and $\mathrm{N}=8$.

Assume a multicast packet arrives in station $s$ and let $\mathcal{D}=\left\{d_{1}, d_{2}, \ldots, d_{k}\right\}$ $\left(d_{1}<d_{2}<\ldots<d_{k}\right)$ denote as the multicast members of this multicast packet. For simplicity, the number of the multicast members is denoted as $|\mathcal{D}|(|\mathcal{D}|=k)$. In BEMA, the way of selecting wavelengths for a multicast packet is divided into three major steps: (1) The BEMA first finds the most shared wavelength (say $w$ ) among these multicast members in set $\mathcal{D}$, which is listened by the most multicast members. The number of multicast members $\left(N M_{i}\right)$ which listen the wavelength i can be calculated by the following equation

$$
N M_{i}=\sum_{j=1}^{|\mathcal{D}|} \sum_{k=1}^{N} f_{s}^{i}\left(k, d_{j}\right),
$$

where

$$
f_{k}^{j}(p, q)= \begin{cases}1, & \text { if } t_{j}(p, q)=k \\ 0, & \text { otherwise }\end{cases}
$$


Therefore, the wavelength $w$ can be easily determined by $w=\left\{k \mid N M_{k} \geq\right.$ $\left.N M_{i}, \forall i, k=1,2, \ldots, C\right\}$. (2) For each multicast member (say $d_{i}$ ), the BEMA checks whether the selected wavelength $\mathrm{w}$ is being used by any of receiver in it. If it does, station $d_{i}$ will be removed from set $\mathcal{D}$ (ie., $\mathcal{D}=\mathcal{D}-\left\{d_{i}\right\}$ ). Otherwise, if there exists any idle receiver in it, an idle receiver will be selected and tuned to this wavelength $w$. Similarly, $d_{i}$ will be removed from set $\mathcal{D}$. (3) If any member is still left in $\mathcal{D}(|\mathcal{D}|>0)$, it will repeat steps (1) and (2) until all multicast member are considered. As mentioned before, if set $\mathcal{D}$ only contains one member, the LBWAA will be applied to further improve the network throughput. This is performed in step (1) to find a proper wavelength instead of finding a random wavelength.

\section{Analysis of BEMA Protocol}

\subsection{Analysis of Random Approach}

Before analyzing the BEMA, we first discuss and analyze the number of transmission times in general cases which using random method to select wavelength/receiver for each multicast packet. In detail, a source station will first select a random wavelength to transmit. If there is any multicast member does not listen to this wavelength, the source station will random select another random wavelength (excluding the wavelength already selected) to cover the remaining stations. This process will be repeated until all multicast members have received the multicast packet successfully. For simplicity, Let $w$ and $k$ denote the total wavelengths and numbers of multicast members of stations in the network, respectively. Moreover, let $t t$ and $t r$ represent the numbers of TTs and TRs equipped in each station, respectively. For the sake of practice, the value of $t r$ is often less than or equal to the total wavelengths $w$.

We analyze all the different combinations and permutations that take just $T$ times to finish a multicast transmission. Now, the number of combination which $k$ multicast members with $\operatorname{tr}$ TRs connect in a network with $w$ wavelengths is

$$
\left(C_{t r}^{w}\right)^{k}
$$

There are two cases to consider for sender taking just $T$ times to finish a multicast transmission. At first, we consider a situation that a multicast member cannot receive any packet before sender transmits the $T$-th transmission. In other word, the receiver did not receive any information at the first $T-1$ transmissions. Let $S(w, t r, T)$ denote the combinations of a multicast member receives packet at the $T$-th transmission successfully. We have

$$
S(w, t r, T)=C_{t r-1}^{w-T}
$$

The second is that one multicast member may receive the packet before the $T$-th transmission. Let $F(w, t r, T)$ denote the combinations of a multicast member receives packet before the $T$-th transmission successfully. This case can 
be considered as a multicast member has $\mathrm{TR}(\mathrm{s})$ which connect to these $(T-1)$ wavelengths that were selected by sender before. Thus, we have

$$
F(w, t r, T)=C_{t r}^{w}-C_{t r}^{w-(T-1)}
$$

For all $k$ multicast members, the number of total combinations of sender transmits $T$ times to complete the transmission can be summed as follows:

$$
\begin{array}{r}
C_{0}^{k} S(w, t r, T)^{0} F(w, t r, T)^{k}+C_{1}^{k} S(w, t r, T)^{1} F(w, t r, T)^{k-1} \\
+\ldots+C_{k}^{k} S(w, t r, T)^{k} F(w, t r, T)^{0}
\end{array}
$$

Substituting Eq. (4) and Eq. (5) into Eq. (6) leads to:

$$
\begin{array}{r}
C_{0}^{k}\left(C_{t r-1}^{w-T}\right)^{0}\left(C_{t r}^{w}-C_{t r}^{w-(T-1)}\right)^{k}+C_{1}^{k}\left(C_{t r-1}^{w-T}\right)^{1}\left(C_{t r}^{w}-C_{t r}^{w-(T-1)}\right)^{k-1} \\
+\ldots+C_{k}^{k}\left(C_{t r-1}^{w-T}\right)^{k}\left(C_{t r}^{w}-C_{t r}^{w-(T-1)}\right)^{0}
\end{array}
$$

which simplifies to

$$
\begin{aligned}
\sum_{i=0}^{k} C_{i}^{k}\left(C_{t r-1}^{w-T}\right)^{i} & \left(C_{t r}^{w}-C_{t r}^{w-(T-1)}\right)^{k-i} \\
& =\left(C_{t r-1}^{w-T}+C_{t r}^{w-T}-C_{t r}^{w-(T-1)}\right)^{k}-\left(C_{t r}^{w}-C_{t r}^{w-(T-1)}\right)^{k}
\end{aligned}
$$

Thus, from Eq. (3) and Eq. (8), the probability $P_{\text {random }}(T)$ of all multicast members receive the packets after sender transmitted $T$ times (with random selecting wavelength) can be computed as follows

$$
P_{\text {random }}(T)=\frac{\left(C_{t r-1}^{w-T}+C_{t r}^{w-T}-C_{t r}^{w-(T-1)}\right)^{k}-\left(C_{t r}^{w}-C_{t r}^{w-(T-1)}\right)^{k}}{\left(C_{t r}^{w}\right)^{k}} .
$$

Since $C_{r}^{n}=C_{r-1}^{n-1}-C_{r}^{n-1}$, we have $C_{t r}^{w-(T-1)}=C_{t r-1}^{w-T}+C_{t r}^{w-T}$. Thus, the probability $P_{\text {random }}(T)$ can be further reduced as

$$
P_{\text {random }}(T)=\frac{\left(C_{t r}^{w}-C_{t r}^{w-T}\right)^{k}-\left(C_{t r}^{w}-C_{t r}^{w-(T-1)}\right)^{k}}{\left(C_{t r}^{w}\right)^{k}} .
$$

Notice that when $T \leq w-t r+1$, the maximum number of transmission times is $w-t r+1$ at the worst case. Now, we can derive the average number of transmission times of a sender to complete a multicast service. That is,

$$
\begin{aligned}
E X P_{\text {random }} & =1 \times P_{\text {random }}(1)+2 \times P_{\text {random }}(2)+\ldots+w \times P_{\text {random }}(w) \\
& =\sum_{i=1}^{w} i \times P_{\text {random }}(i) .
\end{aligned}
$$




\subsection{Analysis of BEMA Approach}

To analysis the BEMA technique, we have to consider the number of TRs. We note that the derived combination cases of a station equipped with one TR and several TRs are quite different. Thus, we divide the conditions into two parts.

A Single Tunable Receiver. The transmission times in BEMA can be considered a Stirling number problem. To arrange $n$ tunable receivers into $r$ wavelengths, we can use $S_{2}(n, r)$ function to calculate the result.

The way to derive all possible onto mapping functions, say $H$, from distribute $k$ distinct stations into $w$ distinct wavelengths can be formalized as the following generating functions. Since no wavelength is empty (by onto definition), each wavelength is listening by at least one station. Therefore, according to the following two equations,

$$
\begin{aligned}
\left(\frac{x}{1 !}+\frac{x^{2}}{2 !}+\frac{x^{3}}{3 !}+\frac{x^{4}}{4 !}+\ldots\right)^{w} & =\left(e^{x}-1\right)^{w} \\
& =\sum_{i=0}^{w} C_{i}^{w}(-1)^{i} e^{(w-1) x}
\end{aligned}
$$

and

$$
\sum_{i=0}^{w} C_{i}^{w}(-1)^{i} \sum_{k=0}^{\infty} \frac{1}{k !}(w-i)^{k} x^{k}=\sum_{k=0}^{\infty} \frac{x^{k}}{k !}\left(\sum_{i=0}^{w}(-1)^{i} C_{i}^{w}(w-i)^{k}\right) .
$$

We can easily derive $H$ by the coefficient of $x^{k} / k$ ! from above equations. Thus, we have

$$
H=\frac{1}{w !}\left(\sum_{i=0}^{w}(-1)^{i} C_{i}^{w}(w-i)^{k}\right) .
$$

Since $S_{2}(k, w)=\frac{1}{w !}\left(\sum_{i=0}^{w}(-1)^{i} C_{i}^{w}(w-i)^{k}\right)$, we have

$$
H=S_{2}(k, w)
$$

From Eq. (15), the probability $P_{B E M A}^{1}(T)$ of all multicast members with one TR receives the multicast packet just after sender transmitted $T$ times can be derived as following:

$$
P_{B E M A}^{1}(T)=\frac{C_{T}^{w} T ! S_{2}(k, T)}{w^{k}} .
$$

Then we can get the expectation $E X P_{B E M A}$ by summering form above $P_{B E M A}^{1}(T)$ : 


$$
\begin{aligned}
\operatorname{EXP}_{B E M A}^{1}(T) & =\frac{1 C_{1}^{w} 1 ! S_{2}(k, 1)+\ldots+w C_{w}^{w} S_{w}(k, w) w !}{w^{k}} \\
& =\frac{\sum_{i=1}^{w} i C_{i}^{w} i ! S_{2}(k, i)}{w^{k}} .
\end{aligned}
$$

In the case of $k<w$, the maximum transmission times of a multicast packet should be less than or equal to $k$. Therefore, we obtain the generalized equation:

$$
\operatorname{EXP}_{B E M A}^{1}(T)=\frac{\sum_{i=1}^{\min (k, w)} i C_{i}^{w} i ! S_{2}(k, i)}{w^{k}} .
$$

The Multi-tunable Receivers. In a multi-tunable receivers model, a station has a chance to connect several channels at a time. Therefore, increasing the number of available receivers will reduce the transmission times. Let $\mathcal{R}=k \cdot t r$ be the total participation multicast members' tunable receivers. And let $\psi$ denote the maximum transmission times of a multicast packet in the case of multiple tunable receivers. Considering the relation between $\mathcal{R}$ and $w$, there are two different cases:

- If $\mathcal{R} \leq w \Rightarrow$ the maximum transmission times will be no more than $\psi=k$ times;

- Otherwise, $\mathcal{R}>w \Rightarrow$ the maximum transmission times will be no more than $\psi=\left\lfloor\frac{w}{t r}\right\rfloor$ times.

First, assume sender only transmits the packet over one wavelength to finish the transmission, all multicast members must listen to a same wavelength. So we can get the combinations as follows:

$$
C_{1}^{w}\left(C_{t r-1}^{w-1}\right)^{k}
$$

From Eq. (19), we want to finish the transmission time at the $T$-th time, the total number of combinations is

$$
\begin{aligned}
C_{1}^{w}\left(C_{t r-1}^{w-1}\right)^{k}-C_{2}^{w}\left(C_{t r-2}^{w-2}\right)^{k}+\cdots & +(-1)^{t r-1} C_{t} r^{w}\left(C_{t r-t r}^{w-t r}\right)^{k} \\
& =\sum_{i=1}^{t r}(-1)^{i-1} C_{i}^{w}\left(C_{t r-i}^{w-i}\right)^{k}
\end{aligned}
$$

Thus, the probability of exact $T$ times is

$$
P_{B E M A}^{t r}(T)=\frac{\sum_{j=T}^{t r}(-1)^{j-T} C_{j}^{w}\left(C_{t r-j}^{w-j}\right)^{k} T ! S_{2}(k, T)}{\left(C_{t r}^{w}\right)^{k}} .
$$


Obviously, from Eq. (21), the expectation value $E X P_{B E M A}^{t r}$ can be obtained by the following equation:

$$
\begin{aligned}
E X P_{B E M A}^{t r} & =1 \times P_{B E M A}^{t r}(1)+2 \times P_{B E M A}^{t r}(2)+\ldots+\psi \times P_{B E M A}^{t r}(\psi) \\
& =\sum_{i=1}^{\psi} i P_{B E M A}^{t r}(i) \\
& =\frac{\sum_{i=1}^{\psi} \sum_{j=i}^{t r} i(-1)^{j-i} C_{j}^{w}\left(C_{t r-j}^{w-j}\right)^{k} i ! S_{2}(k, i)}{\left(C_{t r}^{w}\right)^{k}}
\end{aligned}
$$

\section{Simulation Models and Results}

For simplicity, we assume there are $N$ stations are equally spaced on Bus A. Each station is equipped with a TT and $t r$ TRs. We assume that the distributions of the multicast size and destination stations for all packets are uniform.

To measure the precision of analysis, for each simulation run (100,000 time slots), the average of transmission times is calculated. In the first simulation shown in Fig. 4 we compare the derived analysis result and the simulation result of random approach (RAND) under different number of tunable receivers, different channels and multicast sizes. We can see that the mathematical analyses are very close to the simulation results of RAND approach. Fig. 5 shows the comparisons of the derived analysis result and the simulation result of BEMA approach under different number of tunable receivers, different channels and multicast sizes. We also can see that the analyses are still very close to the simulation results of BEMA approach.

The second simulation is shown in Fig. 6. In this simulation, we investigate how the number of transmission times is affected by the network size. We note that the multicast size $(k)$ is equal to the network size $(N)$. We can see that a larger number of wavelengths or multicast size is, a larger transmission times will obtain. Moreover, we can also find that the analyses are almost matching the derived simulation results.

\section{Conclusion}

In this paper, we analyzed the average transmission times of both random approach (RAND) and best effort multicast approach (BEMA) for providing multicast service over WDM-based multi-channel dual bus networks (MCDBN). Simulations show that the derived analytical results are very close to the simulation results. 


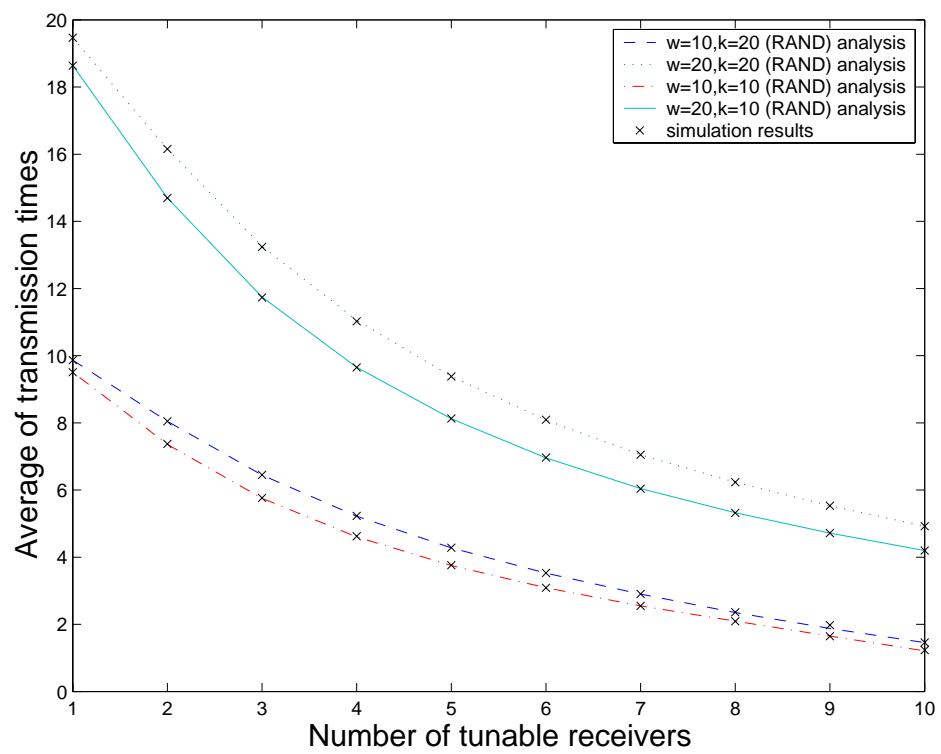

Fig. 4. Comparisons of analysis and simulation results obtained by RAND approach under different number of channels $(w)$, number of tunable receivers $(t r)$ and multicast size $(k)$.

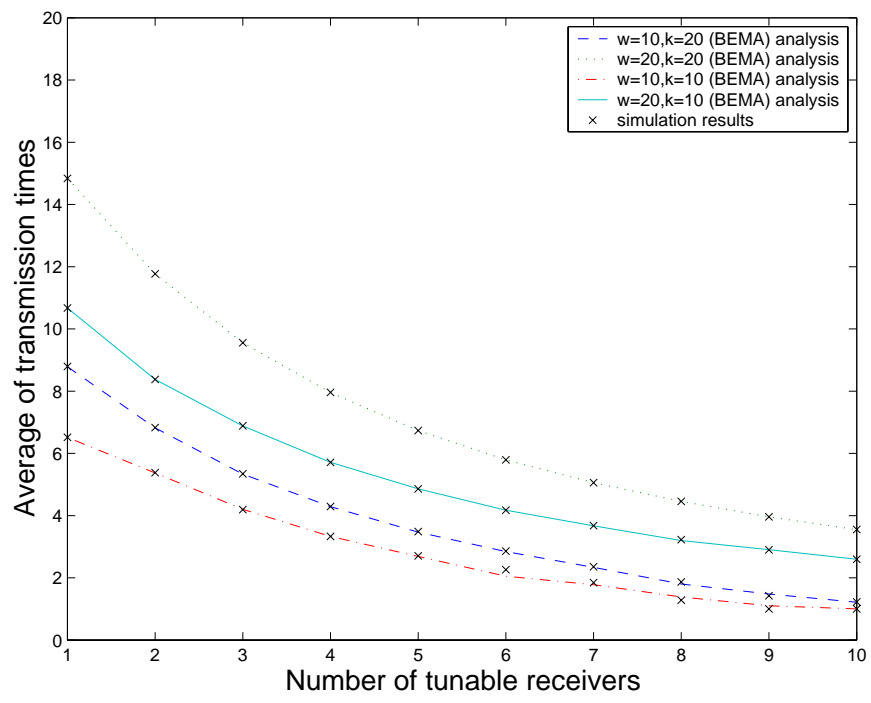

Fig. 5. Comparisons of analysis and simulation results obtained by BEMA approach under different number of channels $(w)$, number of tunable receivers $(t r)$ and multicast size $(k)$. 


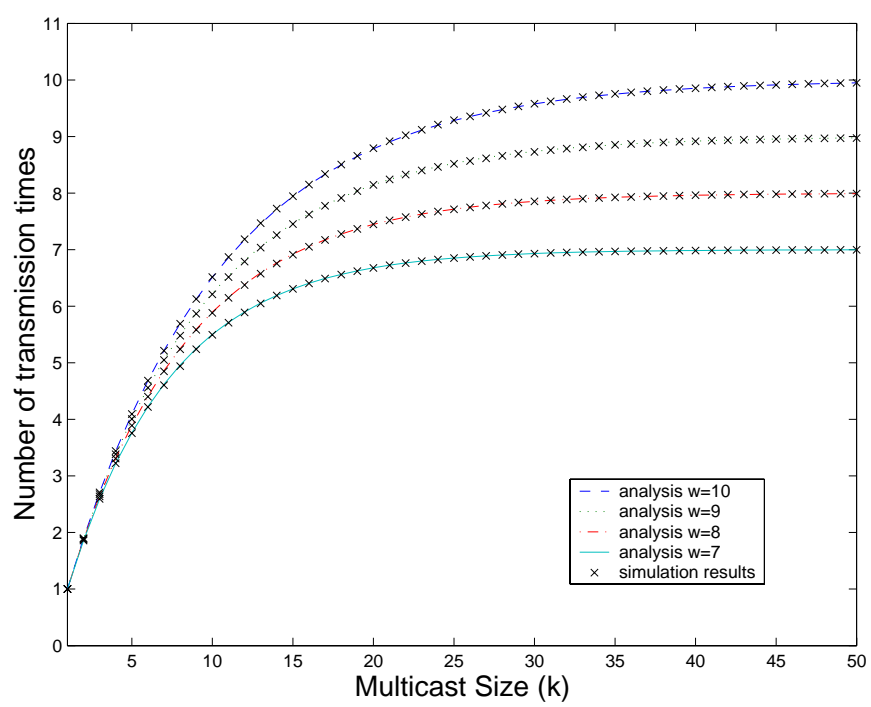

Fig. 6. Comparisons of analysis and simulation results obtained by BEMA approach under different number of channels $(w)$ and multicast size $(k)$ when $t r=1$.

\section{References}

1. S. Banerjee and B. Mukherjee, "An Efficient and Fair Probabilistic Scheduling Protocol for Multi-channel Lightwave Networks," Proc. IEEE SUPERCOMM/ICC'92, Chicago, IL, pp. 1115-1119, May. 1992.

2. C. A. Brackett, "Dense Wavelength Division Multiplexing Networks: Principles and Applications," IEEE J. Select. Areas Commun., vol. 6, no. 8, pp. 948-964, Aug. 1990.

3. K. W. Cheung, "A Subcarrier-Multiplexed Photonic Dual Bus with Tunable Channel Slotted Access for Local/Metropolitan Area Network Applications," in Proc. OFC'92, San Jose, California, pp. FD5:300, Jan. 1992.

4. K. W. Cheung, "Design and Implementation Considerations for WavelengthDivision Multiplexed (WDM) Photonic Dual Bus," Proc. IEEE SUPERCOMM/ICC'92, Chicago, IL, pp. 848-854, May 1992.

5. K. W. Cheung, "EQEB-A Multi-channel Extension of DQDB Protocol with Tunable Channel Access," in Proc. GLOBECOM'92, Orlando, Florida, pp. 1610-1617, Dec. 1992.

6. K. W. Cheung, "Adaptive-Cycle Tunable-Access (ACTA) Protocol: A Simple, High Performance Protocol for Tunable-Channel Multi-Access (TCMA) Networks," Proc. IEEE ICC'93, Geneva, Switzerland, pp. 848-854, Jun. 1993.

7. N. F. Huang and S. T. Sheu, "A slot interleave multiple access scheme for DQDB metropolitan area networks," Proc. IEEE INFOCOM'93, San Francisco, CA, pp. 1075-1082, Mar. 1993.

8. N. F. Huang and S. T. Sheu, "On the Wavelength Assignment Problem of Multichannel Photonic Dual Bus Networks," Proc. IEEE GLOBECOM'94, San Francisco, CA, pp. 1925-1929, Nov. 1994. 
9. N. F. Huang and S. T. Sheu, "DTCAP-A Distributed Tunable-Channel Access Protocol for Multi-Channel Photonic Dual Bus Networks," Proc. IEEE INFOCOM'95, Boston, MASS, pp. 908-915, Apr. 1995.

10. N. F. Huang and S. T. Sheu, "A Wavelength Reusing/Sharing Access Protocol for Multi-Channel Photonic Dual Bus Networks," IEEE J. Lightwave Tech., vol. 14, no. 5, pp. 678-692, May 1996.

11. IEEE P802.6 Working Group, "Proposed standard: Distributed Queue Dual Bus (DQDB) Metropolitan Area Network," approved draft D12, Feb. 1990.

12. Y. M. Lin, D. R. Spears, and M. Yin, "Fiber-based local access network architectures," IEEE Commun. Mag., pp. 64-73, Oct. 1989.

13. J. C. Lu and L. Kleinrock, "A WDMA Protocol for Multichannel DQDB Networks," Proc. IEEE GLOBECOM'93, Houston, TEXAS, pp. 149-153, Nov. 1993.

14. B. Mukherjee, "WDM-based Local Lightwave Networks Part I: Single-Hop Systems," IEEE Network, pp. 12-27, May 1992.

15. B. Mukherjee, "WDM-based Local Local Lightwave Networks Part II: Multi-Hop Systems," IEEE Network, pp. 20-32, Jul. 1992.

16. S. T. Sheu and J. D. Fang, "An Efficient Multicasting Protocol for Multi-channel Dual Bus Networks," Proc. 15th IASTED Int'l Conf. Applied Informatics'97, Innsbruck Austria, pp. 375-378, Feb. 1997. 